Monatsschr Kinderheilkd 2012 • 160:684-684

DOI 10.1007/s00112-012-2712-7

Online publiziert: 5. Juli 2012

(c) Springer-Verlag 2012

Redaktion

G. Hansen, Hannover

R. Kerbl, Leoben

F. Zepp, Mainz

S. Wendt ${ }^{1,2} \cdot{ }^{2}$. Lindenthal ${ }^{3}$

${ }^{1}$ Zentrum für Kinder- und Jugendmedizin, Universitätsmedizin Mainz

2 St. Jude Childrens Research Hospital, Memphis, USA

${ }^{3}$ Hämatologie/Onkologie, Klinik für Allgemeine Kinderheilkunde,

Zentrum für Kinder- und Jugendmedizin, Klinikum Oldenburg

\title{
Pubertas praecox im Kleinkindesalter
}

patoblastomzelltypen, Embryonal- vs. Riesenzellen, produziert. Interessanterweise müssen diese beiden Tumorzelltypen im Rezidiv oder Metastasengewebe nicht gemeinsam vorkommen. Damit sind $\beta$-HCG oder Testosteron, wie von der Autorin angeregt, nicht besonders gut als Verlaufsmarker nach vollständiger Remission geeignet $[1,3,4]$.

\section{S. Wendt}

St. Jude Childrens Research Hospital, Memphis, USA

Gerne möchte ich den oben genannten Artikel kommentieren, da mir einige Fakten der Diskussion ergänzungswürdig erscheinen, und es schade wäre, wenn junge Assistenten oder Fachärzte, die nicht im Fachgebiet der Onkologie tätig sind, unvollständige und dadurch $\mathrm{m}$. E. fehlerhafte Informationen erhielten.

Aus dem Artikel lässt sich missverständlich herauslesen, dass Hepatoblastome Testosteron produzieren. Das ist allerdings nicht ganz korrekt. Die paraneoplastische Pubertas praecox entsteht durch die Produktion von $\beta$-HCG (humanes Choriongonadotropin $\beta$ ) im Tumorgewebe, welches wiederum die Leydig-Zellen im Hoden zur Testosteronproduktion anregt $[2,3]$.

$\beta$-HCG ist ein Heterodimer, dessen $\alpha$ Untereinheit der des LH (luteinisierendes Hormon) gleicht. Interessanterweise findet sich meist keine signifikante Vergrößerung der Hoden, weil keine Spermatogenese stattfindet, die FSH-abhängig (FSH: Follikel stimulierendes Hormon) ist. Aus dem gleichen Grund weisen Mädchen mit autonomer $\beta$-HCG-Sekretion meist keine Pubertätsanzeichen auf, da hierfür FSH erforderlich wäre.

AFP ( $\alpha$-Fetoprotein), der typische Tumormarker für Hepatoblastom, der in $>90 \%$ dieser Fälle gebildet wird, und $\beta$ HCG werden von unterschiedlichen $\mathrm{He}$ -

\section{Korrespondenzadresse}

\section{Dr. S. Wendt}

Fellow Hematology/Oncology Dept.

St. Jude Children's Research Hospital

262 Danny Thomas Place MS 260

Memphis, TN 38105

susanne.wendt@stjude.org

\section{Literatur}

1. Braunstein GD, Bridson WE, Glass A et al (1972) In vivo and in vitro production of human chorionic gonadotropin and alpha-feteoprotein by a virilizing hepatoblastoma. J Clin Endocrinol Metab 35(6):857-862

2. Kumar EV, Kumar L, Pathak IC et al (1978) Clinical, hormonal and ultrastructure studies of a virilizing hepatoblastoma. Acta Paediatr Scand 67(3):389-392

3. Nakagawara A, Ikeda K, Tsuneyoshi M et al (1985) Hepaman chorionic gonadotropin. Clinicopathologic analysis of four cases and a review of the literature. Cancer 56(7):1636-1642

4. Navarro C, Corretger JM, Sancho A et al (1985) Paraneoplasic precocious puberty. Report of a new case with hepatoblastoma and review of the literature. Cancer 56(7):1725-1729

\section{Erwiderung}

\section{Lindenthal}

Zentrum für Kinder- und Jugendmedizin,

Klinikum Oldenburg

Herzlichen Dank für den interessanten Kommentar zu unserer Fallvorstellung des Patienten mit einer hepatoblastombedingten Pubertas praecox! Tatsächlich wiesen wir nicht explizit darauf hin, dass die tumorbedingte Testosteronerhöhung toblastoma producing both alpha-fetoprotein and hu- via $\beta$-HCG zustande kommt und nicht der Tumor selbst Testosteron produziert, was Sie wertvoller Weise klarstellen.

Selbstverständlich sind Verlaufskontrollen von Androgenen und $\beta$-HCG in der onkologischen Nachsorge kein Garant für die frühzeitige Erkennung eines Rezidivs, da dieses - wie von Ihnen dargestellt - abweichend von der Ersterkrankung nicht mit einer Erhöhung dieser Hormone einhergehen muss. Deshalb wiesen wir lediglich auf den wertvollen Beitrag, welchen diese Parameter als zusätzliche Tumormarker leisten können, hin, ohne im Umkehrschluss ein Rezidiv bei fehlender Erhöhung ausschließen oder andere Nachsorgeuntersuchungen vernachlässigen zu wollen.

\section{Korrespondenzadresse}

\section{Dr. V. Lindenthal}

Hämatologie/Onkologie,

Klinik für Allgemeine Kinderheilkunde

Zentrum für Kinder- und Jugendmedizin,

Klinikum Oldenburg

Rahel-Straus-Straße 10

26133 Oldenburg

violalindenthal@googlemail.com

\section{Literatur}

1. Braunstein GD, Bridson WE, Glass A et al (1972) In vivo and in vitro production of human chorionic gonadotropin and alpha-feteoprotein by a virilizing hepatoblastoma. J Clin Endocrinol Metab 35(6):857-862

2. Kumar EV, Kumar L, Pathak IC et al (1978) Clinical, hormonal and ultrastructure studies of a virilizing hepatoblastoma. Acta Paediatr Scand 67(3):389-392

3. Nakagawara A, lkeda K, Tsuneyoshi M et al (1985) Hepatoblastoma producing both alpha-fetoprotein and human chorionic gonadotropin. Clinicopathologic analysis of four cases and a review of the literature. Cancer 56(7):1636-1642

4. Navarro C, Corretger JM, Sancho A et al (1985) Paraneoplasic precocious puberty. Report of a new case with hepatoblastoma and review of the literature. Cancer 56(7):1725-1729 